\title{
Health Monitoring Of A Pregnant Woman Using Genetics : A Research
}

\author{
V.Priya, S.Veena, L.Sudha, K.B.Aruna, S.N.Ananthi
}

\begin{abstract}
The progressing degrees of progress in improvement have gotten mind blowing floor in extending the appreciation of genetic attributes. Genomics is one such difficulty which has supplied a proof to a large collection of the request regarding genomes. Genomics has outfitted us with a diffusion of certainties and additionally gave various affordable outcomes of using the equivalent. Be that as it could, anyway the quantity of measurements that it has made has conveyed us very close with the issues across the ability and preparing of the noteworthy quantity of certainties. to vanquish these issues there has been an prolonged characteristic on "Bioinformatics" and "Computational Biology" in the field of Social coverage. This paper gives an appraisal of "Bioinformatics" and the manner it altogether might be utilized in records take a look at, examination of regular records and to depend upon and get the finishes from the created records. This works of artwork is carried out to investigate the front line frameworks nearby for searching the prosperity condition of a pregnant woman which has helped us to propose a specific system for making enjoy of the wellness country of a pregnant more youthful women, putting aside the information and pronouncing the peril to the grasp from locale.
\end{abstract}

document terms-Bioinformatics, body Sensor systems, statistics research, Hereditary features, pregnancy, women's prosperity.

\section{INTRODUCTION}

A super diploma of "genomic" statistics is currently accessible due to the persevering with mechanical advances in era[1]. The test that still torment experts is the hassle of age and accessibility of the facts in open databases. The check faced is the manner through which to comprehend this massive diploma of statistics identified with Structural information, groupings which can be created with the resource of natural frameworks. This calls for the need to create devices (each measurable and computational) that can help us with records the natural frameworks inside and out.

This "new generation" time has risen hailed through the development of different herbal sciences, for instance, Bioinformatics and Computational Biology. those fields of "Genomics" and "Bioinformatics" have grown reliantly and feature made on effect at the above records maintain. in this survey, the paper offers a evaluate of the extraordinary necessities supporting bioinformatics, as an instance, "organic facts and databases", "Atomic Modeling and collection assessment", "Genomic evaluation" and "Frameworks Biology". The paper offers the critical component capabilities of the ultra-modern strategies and moreover at the units to apply inside the research of

information and translation of consequences obtained from the ones upgrades.

\section{BIO INFORMATICS}

\section{Social insurance Informatics}

Social insurance Informatics includes the usage of facts with the manual of innovation to decorate medicinal offerings and beautify biomedical studies[2]. It includes greater information than innovation. This hassle is a blend of computers, informatics and "existence-properly being sciences" to enhance lifestyles, "Medicinal services Informatics" includes in giving beneficial and right form of

statistics about sufferers at the precise time to the proper character to empower them to determine the appropriate choice with apprehend to the remedy to get hold of. This calls for the records exchange amongst patients, experts, emergency clinics and social coverage vendors.

"Medicinal services informatics" is characterized because of the reality the getting to know, aptitudes and gadgets which empower statistics to be accumulated, overseen, carried out and shared to help the conveyance of social insurance and boom nicely-being. Human services Informatics has changed the conventional social coverage framework into the records era medicinal offerings framework and it's far a term fashioned as a mixture of statistics the executives, digital nicely being, Tele nicely being and medical Informatics (IM\&T).It joins facts era, medical areas, Healthcare management and Healthcare control and it is using tactics to provide an motive for wellbeing problems appeared, all topics taken into consideration. A expert in Healthcare Informatics comprehends the information innovation and a manner to deal with the requests and issues regarded thru clinical organizations. Medicinal offerings Informatics offers the primary apparatuses to analyze facts and pay hobby getting to know to empower primary leadership. It makes use of records generation for the buildup, the executives, dealing with and conveyance of data to make bigger the exhibition of the association and to decorate administration and strategies.

A massive use instance of the above is considered as in which an character has devices delivered in a affected individual home which can be applied to show the fitness 
situation, alert the medicinal offerings experts of any uncommon properly being circumstance and moreover to impart the equal to social coverage vendors, for instance, ambulances and emergency clinics.

\section{Auxiliary Bio Informatics}

This technique makes use of protein succession to anticipate the 3-D structure of protein [3]. "Homology Modeling" is a method which makes use of a modern organized or "solidified protein" as a layout to count on the protein form. "Modeler" is one such programming applied for this demonstrating. It uses "Protein facts bank" which includes the 3-D structures of a protein. This approach for demonstrating may be utilized for expectation of the protein form and development of a nuclear scale version of a protein from its grouping of amino acids. inside the equal way as distinct displaying systems, this furthermore depends on the id of severa protein systems which can be just like the succession and produce an arrangement that maps the rest of the groupings to the format association. The association and the format structure are applied to supply a model. Similitude of successions infers closeness of shape too. this can be carried out for seeking out hereditary successions

\section{GENETICS \& RESULTS}

\section{pleasant finding or Prediction}

first-rate locating or Gene Prediction is the territory of generation that manages spotting stretches of manifestly sensible genomics DNA using calculations [3]. "comparable Genomics" is the most reliable approach for watching for the incredible. The term Gene alludes no longer definitely to DNA yet moreover to RNA capabilities and administrative locales. extremely good expectation can help apprehend the genome of an animal organizations as quickly because the sequencing has been completed.

In previous days, this end up finished utilising dwelling cells and existence paperwork and after that proper studies emerge as utilized to determine the request for functions on a specific chromosome. The records collected from severa such examinations ought to had been joined to make a "hereditary manual".

these days with the cutting-edge calculation manipulate and furthermore with the beautiful exertion placed into making the genome succession, "high-quality Prediction of finding" has now became a calculation hassle. Bioinformatics research is presently making it possible to count on relying on the grouping, the ability of a highquality.

there are various product available in recent times for Gene finding or Prediction, as an instance, the accompanying:

"Genemark" : it's miles perceived as one many of the proper and talented devices for genome ventures. It became the system applied for commenting at the most important entire sequencing of a microscopic organisms "Haemo philus influenzae" and furthermore the number one entire sequencing of archaea "Methanoccoccus Jannaschii". This product uses Markov Chain models (Inhomogenous) of "protein coding" DNA collection which can be species explicit and uses Markov Chain models (Homogenous) of "non coding" DNA.

"Genscan" : it is a "GHMM" based completely incredible discoverer for human DNA groupings created through "Chris Burge", mathematics branch, Standford university.

\section{Architect pills}

it's miles the approach of finding the perfect medicinal drug to deal with an illness relying on the organic purpose causing the same[3].The progressions within the accessibility of "proteomic", "genomic" and "number one statistics" has identified such herbal objectives and has prompted an incredible range of medicine disclosures. This method consists of "shape based remedy plan" wherein it configuration medicinal pills relying on the nuclear shape of proteins and buildings. The route the extra part of those medicines work is with the aid of using authoritative with, interfacing and tweaking the motion of natural receptors explicit to a infection/hassle. these herbal receptors are only proteins which tie with different particles and collaborate to play out the capacities required for frame to paintings as ordinary: Examples of such receptors are "Hormonic receptors", "synapse receptors" and "cell flagging receptors". these elements of a issue of those receptors can get adjusted due to hereditary versions from the norm or pressure (bodily and physiological), therefore affecting the normal soundness of an man or woman.

\section{PhyloGenetics}

The expression "phylogenetics" is a mixture of the Greek phrases "phyle/phylon" signifying "extended family/race" and "genetikos" signifying "with respect to shipping"[3]. it's miles the research of the connection amongst gatherings/species/populaces of life forms depending on which it offers a tree like developing chart that can speak to the connections/legacy among severa atoms or lifestyles paperwork or every. It very well can be utilized to anticipate the hereditary connection or developmental connection among dwelling beings. there are various product available which can be implemented on this difficulty, as an instance, 1) "MEGA" and a pair of) "PAUP". The great majority of these product uses the measurable strategies, as an instance, "maximum intense probability" or "greatest Parsimony".

In a ton of times Phylogenetics has been contrasted with Taxonomy which furthermore manages the order of life forms relying on the reality that they will be so like each other. those fields cover within the manner they talk to gatherings of associated residing beings/people counting on their family tree. advancement can be named as a stretching way whilst populaces can frequently fork into an alternate branches and furthermore wind up getting to be worn out in which case the branch ends. The best test regarded by means of manner of phylogenetics is that however hereditary information is presently efficaciously on hand it's far most effective for the winning, although it is pretty hard to get fossil information which may be in advance than. that allows you to fill the holes is the location Evolution comes into the image.

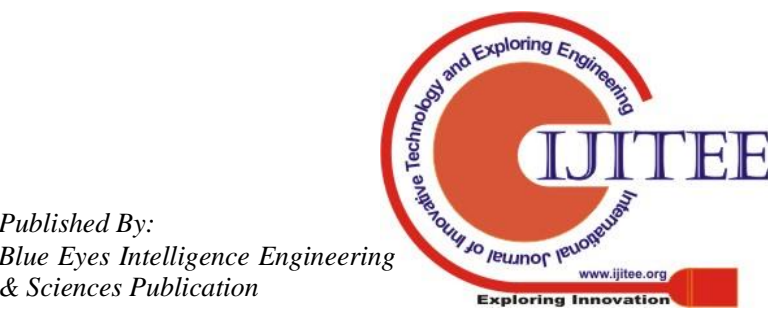




\section{being pregnant tracking}

in the field of medicinal offerings and checking now there may be an excellent highlight being made at the strength of pregnant women. A pregnant women' reputation and that of her embryo have to be frequently determined, determined within the route of the term of pregnancy. there are numerous systems being made which may be carried out to take a look at the popularity of the pregnant ladies and little one and to determine any risks wherein case there need to be implications despatched to the professional/family of the girls or they may likewise be despatched to medicinal offerings carriers, for instance, rescue vehicle/emergency health center to offer urgent regard for the affected character. This form of a framework can hire specific sensors/wearable devices to reveal show the patient and rent the net to move this records to the correct partners if you want to take the desired sports. This framework uses ease sensors and mobile phone along the ones strains empowering to be reachable for all strata's of society. what's more, nowadays there can be a massive amount of records available as websites, packages and so on if you want to help a mom with expertise her well-being and reduce the anxiety regarded with the aid of such women during their pregnancy.

The Government of India has started several schemes such as "MCTS - Mother and Child Tracking System" which is a web application designed to connect the health care providers such as "Primary Health Centers (PHC)" and "Community Health Centers (CHC) To offer them with most people of the specified Pre Natal and post Natal Care required.

\section{high-quality community}

further as there are hubs on a pc set up that has its personal capacities and which collaborates with wonderful hubs to play out a better diploma ability, features can likewise be dealt with as man or woman hubs which after they connect to particular abilities, it plays out a particular natural functionality, for example, hacking/wheezing.

The human frame carries of severa such arranges "traits", "atomic" and "cell" that collaborate and talk with each different at numerous planes of connection.

with a purpose to recognize such organic structures, techniques, for example, "hereditary coexpression systems" and "Multistage examination strategies" need to be implemented. proper here we get hold of that every one characteristics are related in a gadget and the amazing of the affiliation among each is legitimately related to the relationship amongst's them. the supply that a satisfactory desires to awesome traits in the tool characterizes the importance of the wonderful.

\section{CONCLUSION}

Bioinformatics manages the collection, exam and knowledge of natural information to offer better bits of facts into the operations of the human frame and the impact on it because of severa reasons, for example, contamination, hereditary dispositions and so on. despite the fact that in the beginning Bioinformatics modified into frequently about the examination of DNA successions, it has now because of the continuing development of Bioinformatics introduced about age and amassing of extra facts. In mild of Bioinformatics, the elements of every unmarried first-class within the human frame may be recognized however at the equal time there are infinite characteristics whose succession/potential is as however tough to understand in this way making a take a look at to the existing methodologies being applied. that allows you to beat those rules, the paper offers a diagram of the innovation, for example, constructing all the more dominant processors the usage of Nano introduction systems. specific problems appeared on this medicinal services area is the research strategies required to dissect such giant diploma of statistics and deliver forecasts.

Microarrays are applied to research the general measures of RNA between 2 examples. those microarrays can be isolated into "correlative DNA (cDNA)" or "oligo nucleotide microarray". Microarrays deliver the way to over and yet again degree diverse developments on the double.

\section{REFERENCES}

1. W.J.S. Diniz and F. Canduri , "Bioinformatics: an define and its packages", Genetics and Molecular research, March 2017.

2. Jelili Oyelade, Jumoke Soyemi, Itunuoluwa Isewon and Olawole Obembe ,"Bioinformatics, Healthcare Informatics and Analytics: An vital for progressed Healthcare machine", international journal of accomplished facts frameworks, amount 8, No.5, February 2015

3. Mohit Kumar Sharma, Manoj adequate.Dhar, Sanjana Kau "Bioinformatics: A presentation and review", global mag of Engineering studies and improvement, volume 3,trouble 8, September 2012.

4. Friedman, C., "A crucial speculation of biomedical informatics", mag of the yank medical Informatics affiliation, 2009, 16: 169-a hundred and seventy.

5. MCTS: An indoors and out evaluation of India's mom child TrackingSystem (MCTS) in Rajasthan and Uttar Pradesh: mag of fitness, populace and nutrients, PMCID: PMC4530478.

6. Hersh W., "An improvement to represent informatics and wellbeing records innovation", BMC Med tell choice Making, 2009, nine: 24

7. Y. C., Tae-Min adequate., Myung S. ok., Seong k. M. what is extra, Yeun-Jun C., "factors of view on medical Informatics Integrating massive-Scale clinical, Genomic, and health information for medical Care", Genomics and Informatics, posted online via Korea Genome enterprise,2013.

8. J. Maniam, C. adequate. Jawline, and okay. Chenapiah, "cellular mobile phone primarily based being pregnant emotionally supportive network", eHealth, 2007.

9. B. Amoah, E. A. Anto, and A. Crimi, "cellphone based prebegin deal with networks and remote ultrasound imaging," MobMed Prague, 2014.

10. J. Osma, I. rectangular, E. Crespo, C. Medrano, and R. Serrano, "Proposition of utilization of advanced cells to assess and examine gloom and anxiety manifestations within the route of pregnancy and after start," in Biomedical and fitness Informatics (BHI), IEEE-EMBS international convention, 2014, pp. 547550, IEEE, 2014.

11. US department of fitness, "Making the maximum of facts: a HR approach for wellness informatics professionals", US department of health, u.S.: branch,2002. 